\title{
RESEARCH
}

\section{Cracking the problem with 33}

\author{
Andrew R. Booker(i)
}

*Correspondence:
andrew.booker@bristol.ac.uk
School of Mathematics,
University of Bristol, University
Walk, Bristol BS8 1TW, UK
This work was carried out using
the computational facilities of
the Advanced Computing
Research Centre, University of
Bristol, http://www.bris.ac.uk/
acrc/. The author was partially
supported by EPSRC Grant
EP / K034383 / 1. No data were
created in the course of this
study.

${ }^{*}$ Correspondence:

andrew.booker@bistol.ac.uk School of Mathematics, University of Bristol, University This work was carried out using Bristol, http://www bris.ac uk/ acrc/. The author was partially created in the course of this

\begin{abstract}
Inspired by the Numberphile video "The uncracked problem with 33" by Browning and Brady Haran (https://youtu.be/wymmCdLdPvM), we investigate solutions to

$x^{3}+y^{3}+z^{3}=k$ for a few small values of $k$. We find the first known solutions for $k=33$ and $k=795$.

Keywords: Sums of three cubes, Diophantine equations, Hasse principle
\end{abstract}

\section{Introduction}

Let $k$ be a positive integer with $k \not \equiv \pm 4(\bmod 9)$. Then Heath-Brown [9] has conjectured that there are infinitely many triples $(x, y, z) \in \mathbb{Z}^{3}$ such that

$$
k=x^{3}+y^{3}+z^{3} \text {. }
$$

Various numerical investigations of (1) have been carried out, beginning as early as 1954 [13]; see [1] for a thorough account of the history of these investigations up to 2000 . The computations performed since that time have been dominated by an algorithm due to Elkies [6]. The latest that we are aware of is the paper of Huisman [11] (based on the implementation by Elsenhans and Jahnel [7]), which determined all solutions to (1) with $k<1000$ and $\max \{|x|,|y|,|z|\} \leq 10^{15}$. In particular, Huisman reports that solutions are known for all but 13 values of $k<1000$ :

$$
\text { 33, 42, 114, 165, 390, 579, 627, 633, 732, 795, 906, 921, } 975 .
$$

Elkies' algorithm works by finding rational points near the Fermat curve $X^{3}+Y^{3}=1$ using lattice basis reduction; it is well suited to finding solutions for many values of $k$ simultaneously. In this paper we describe a different approach that is more efficient when $k$ is fixed. It has the advantage of provably finding all solutions with a bound on the smallest coordinate, rather than the largest as in Elkies' algorithm. This always yields a nontrivial expansion of the search range since, apart from finitely many exceptions that can be accounted for separately, one has

$$
\max \{|x|,|y|,|z|\}>\sqrt[3]{2} \min \{|x|,|y|,|z|\} .
$$

Moreover, empirically it is often the case that one of the variables is much smaller than the other two, so we expect the gain to be even greater in practice.

Our strategy is similar to some earlier approaches (see especially $[3,10,12]$ and [1]), and is based on the observation that in any solution, $k-z^{3}=x^{3}+y^{3}$ has $x+y$ as a factor.

(c) The Author(s) 2019. This article is distributed under the terms of the Creative Commons Attribution 4.0 International License (http://creativecommons.org/licenses/by/4.0/), which permits unrestricted use, distribution, and reproduction in any medium, provided you give appropriate credit to the original author(s) and the source, provide a link to the Creative Commons license, and indicate if changes were made. 
Our main contribution over the earlier investigations is to note that with some time-space tradeoffs, the running time is very nearly linear in the height bound, and it is quite practical when implemented on modern 64-bit computers.

In more detail, suppose that $(x, y, z)$ is a solution to (1), and assume without loss of generality that $|x| \geq|y| \geq|z|$. Then we have

$$
k-z^{3}=x^{3}+y^{3}=(x+y)\left(x^{2}-x y+y^{2}\right) .
$$

If $k-z^{3}=0$ then $y=-x$, and every value of $x$ yields a solution. Otherwise, setting $d=|x+y|=|x|+y \operatorname{sgn} x$, we see that $d$ divides $\left|k-z^{3}\right|$, and

$$
\begin{aligned}
\frac{\left|k-z^{3}\right|}{d} & =x^{2}-x y+y^{2}=x(2 x-(x+y))+y^{2} \\
& =|x|(2|x|-d)+(d-|x|)^{2}=3 x^{2}-3 d|x|+d^{2},
\end{aligned}
$$

so that

$$
\{x, y\}=\left\{\frac{1}{2} \operatorname{sgn}\left(k-z^{3}\right)\left(d \pm \sqrt{\frac{4\left|k-z^{3}\right|-d^{3}}{3 d}}\right)\right\} .
$$

Thus, given a candidate value for $z$, there is an effective procedure to find all corresponding values of $x$ and $y$, by running through all divisors of $\left|k-z^{3}\right|$. Already this basic algorithm finds all solutions with $\min \{|x|,|y|,|z|\} \leq B$ in time $O\left(B^{1+\varepsilon}\right)$, assuming standard heuristics for the time complexity of integer factorization. In the next section we explain how to avoid factoring and achieve the same ends more efficiently.

\section{Methodology}

For ease of presentation, we will assume that $k \equiv \pm 3(\bmod 9)$; note that this holds for all $k$ in (2). Since the basic algorithm described above is reasonable for finding small solutions, we will assume henceforth that $|z|>\sqrt{k}$. Also, if we specialize (1) to solutions with $y=z$, then we get the Thue equation $x^{3}+2 y^{3}=k$, which is efficiently solvable. Using the Thue solver in PARI/GP [18], we verify that no such solutions exist for the $k$ in (2). Hence we may further assume that $y \neq z$.

Since $|z|>\sqrt{k} \geq \sqrt[3]{k}$, we have

$$
\operatorname{sgn} z=-\operatorname{sgn}\left(k-z^{3}\right)=-\operatorname{sgn}\left(x^{3}+y^{3}\right)=-\operatorname{sgn} x .
$$

Likewise, since $x^{3}+z^{3}=k-y^{3}$ and $|y| \geq|z|$, we have $\operatorname{sgn} y=-\operatorname{sgn} x=\operatorname{sgn} z$. Multiplying both sides of (1) by $-\operatorname{sgn} z$, we thus obtain

$$
|x|^{3}-|y|^{3}-|z|^{3}=-k \operatorname{sgn} z .
$$

Set $\alpha=\sqrt[3]{2}-1$, and recall that $d=|x+y|=|x|-|y|$. If $d \geq \alpha|z|$ then

$$
\begin{aligned}
-k \operatorname{sgn} z & =|x|^{3}-|y|^{3}-|z|^{3} \geq(|y|+\alpha|z|)^{3}-|y|^{3}-|z|^{3} \\
& =3 \alpha(\alpha+2)(|y|-|z|) z^{2}+3 \alpha(|y|-|z|)^{2}|z| \\
& \geq 3 \alpha(\alpha+2)|y-z| z^{2} .
\end{aligned}
$$

Since $3 \alpha(\alpha+2)>1$, this is incompatible with our assumptions that $y \neq z$ and $|z|>\sqrt{k}$. Thus we must have $0<d<\alpha|z|$. 
Next, reducing (4) modulo 3 and recalling our assumption that $k \equiv \pm 3(\bmod 9)$, we see that

$$
d=|x|-|y| \equiv|z| \quad(\bmod 3) .
$$

Let $\epsilon \in\{ \pm 1\}$ be so that $k \equiv 3 \epsilon(\bmod 9)$. Then, since every cube is congruent to 0 or $\pm 1(\bmod 9)$, we must have $x \equiv y \equiv z \equiv \epsilon(\bmod 3)$, so that $\operatorname{sgn} z=\epsilon\left(\frac{|z|}{3}\right)=\epsilon\left(\frac{d}{3}\right)$. In view of (3), we get a solution to (1) if and only if $d \mid z^{3}-k$ and $3 d\left(4\left|z^{3}-k\right|-d^{3}\right)=$ $3 d\left(4 \epsilon\left(\frac{d}{3}\right)\left(z^{3}-k\right)-d^{3}\right)$ is a square.

In summary, to find all solutions to (1) with $|x| \geq|y| \geq|z|>\sqrt{k}, y \neq z$ and $|z| \leq B$, it suffices to solve the following system for each $d \in \mathbb{Z} \cap(0, \alpha B)$ coprime to 3 :

$$
\begin{aligned}
& \frac{d}{\sqrt[3]{2}-1}<|z| \leq B, \quad \operatorname{sgn} z=\epsilon\left(\frac{d}{3}\right), \quad z^{3} \equiv k \quad(\bmod d), \\
& 3 d\left(4 \epsilon\left(\frac{d}{3}\right)\left(z^{3}-k\right)-d^{3}\right)=\square
\end{aligned}
$$

Our approach to solving this is straightforward: we work through the values of $d$ recursively by their prime factorizations, and apply the Chinese remainder theorem to reduce the solution of $z^{3} \equiv k(\bmod d)$ to the case of prime power modulus, to which standard algorithms apply. Let $r_{d}(k)=\#\left\{z(\bmod d): z^{3} \equiv k(\bmod d)\right\}$ denote the number of cube roots of $k$ modulo $d$. By standard analytic estimates, since $k$ is not a cube, we have

$$
\sum_{d \leq \alpha B} r_{d}(k) \ll_{k} B
$$

Heuristically, computing the solutions of $z^{3} \equiv k(\bmod p)$ for all primes $p \leq \alpha B$ can be done with $O(B)$ arithmetic operations on integers in $[0, \alpha B]$; see e.g. the algorithm described in [17, Sect. 2.9, Exercise 8]. Assuming this, one can see that with Montgomery's batch inversion trick [15, Sect. 10.3.1], the remaining effort to determine the roots of $z^{3} \equiv k(\bmod d)$ for all positive integers $d \leq \alpha B$ can again be carried out with $O(B)$ arithmetic operations.

Thus, we can work out all $z$ satisfying the first line of (5), as a union of arithmetic progressions, in linear time. To detect solutions to the final line, it is crucial to have a quick method of determining whether $\Delta:=3 d\left(4 \epsilon\left(\frac{d}{3}\right)\left(z^{3}-k\right)-d^{3}\right)$ is a square. We first note that for fixed $d$ this condition reduces to finding an integral point on an elliptic curve; specifically, writing $X=12 d|z|$ and $Y=(6 d)^{2}|x-y|$, from (3) we see that $(X, Y)$ lies on the Mordell curve

$$
Y^{2}=X^{3}-2(6 d)^{3}\left(d^{3}+4 \epsilon\left(\frac{d}{3}\right) k\right) .
$$

Thus, for fixed $d$ there are at most finitely many solutions, and they can be effectively bounded. For some small values of $d$ it is practical to find all the integral points on (6) and check whether any yield solutions to (1). For instance, using the integral point functionality in Magma [2, Sect. 128.2.8], we verified that there are no solutions for $k$ as in (2) and $d \leq 40$, except possibly for $(k, d) \in\{(579,29),(579,34),(975,22)\}$.

Next we note that some congruence and divisibility constraints come for free:

Lemma Let $z$ be a solution to (5), let $p$ be a prime number, and set $s=\operatorname{ord}_{p} d, t=$ $\operatorname{ord}_{p}\left(z^{3}-k\right)$. Then: 
(i) $z \equiv \frac{4}{3} k\left(2-d^{2}\right)+9(k+d)(\bmod 18)$;

(ii) if $p \equiv 2(\bmod 3)$ then $t \leq 3 s$;

(iii) if $t \leq 3 s$ then $s \equiv t(\bmod 2)$;

(iv) if $\operatorname{ord}_{p} k \in\{1,2\}$ then $s \in\left\{0, \operatorname{ord}_{p} k\right\}$.

Proof Let $\Delta=3 d\left(4 \epsilon\left(\frac{d}{3}\right)\left(z^{3}-k\right)-d^{3}\right)$. Writing $\delta=\left(\frac{d}{3}\right)$, we have $|z| \equiv d \equiv \delta$ $(\bmod 3)$. Observing that $(\delta+3 n)^{3} \equiv \delta+9 n(\bmod 27)$, modulo 27 we have

$$
\begin{aligned}
\frac{\Delta}{3 d} & =4 \epsilon \delta\left(z^{3}-k\right)-d^{3}=4|z|^{3}-d^{3}-4 \epsilon \delta k \\
& \equiv 4[\delta+3(|z|-\delta)]-[\delta+3(d-\delta)]-4 \epsilon \delta k=3(4|z|-d)-\delta[18+4(\epsilon k-3)] \\
& \equiv 3(4|z|-d)-d[18+4(\epsilon k-3)]=12|z|-9 d-4 \epsilon d k \\
& \equiv 3|z|-4 \epsilon d k .
\end{aligned}
$$

This vanishes modulo 9 , so in order for $\Delta$ to be a square, it must vanish mod 27 as well. Hence

$$
z=\epsilon \delta|z| \equiv \frac{4 \delta d k}{3} \equiv \frac{4\left(2-d^{2}\right) k}{3} \quad(\bmod 9) .
$$

Reducing (1) modulo 2 we see that $z \equiv k+d(\bmod 2)$, and this yields (i).

Next set $u=p^{-s} d$ and $v=p^{-t} \epsilon \delta\left(z^{3}-k\right)$, so that

$$
\Delta=3\left(4 p^{s+t} u v-p^{4 s} u^{4}\right) .
$$

If $3 s<t$ then $p^{-4 s} \Delta \equiv-3 u^{4}(\bmod 4 p)$, but this is impossible when $p \equiv 2(\bmod 3)$, since -3 is not a square modulo $4 p$. Hence we must have $t \leq 3 s$ in that case.

Next suppose that $t \leq 3 \mathrm{~s}$. We consider the following cases, which cover all possibilities:

- If $p=3$ then $s=t=0$, so $s \equiv t(\bmod 2)$.

- If $p \neq 3$ and $3 s>t+2 \operatorname{ord}_{p} 2$ then $\operatorname{ord}_{p} \Delta=s+t+2 \operatorname{ord}_{p} 2$, so $s \equiv t(\bmod 2)$.

- If $3 s \in\{t, t+2\}$ then $s \equiv t(\bmod 2)$.

- If $p=2$ and $3 s=t+1$ then $2^{-4 s} \Delta=3\left(2 u v-u^{4}\right) \equiv 3(\bmod 4)$, which is impossible.

Thus, in any case we conclude that $s \equiv t(\bmod 2)$.

Finally, suppose that $p \mid k$ and $p^{3} \nmid k$. If $s=0$ then there is nothing to prove, so assume otherwise. Since $d \mid z^{3}-k$, we must have $p \mid z$, whence

$$
0<s \leq t=\operatorname{ord}_{p}\left(z^{3}-k\right)=\operatorname{ord}_{p} k<3 s .
$$

By part (iii) it follows that $s \equiv \operatorname{ord}_{p} k(\bmod 2)$, and thus $s=\operatorname{ord}_{p} k$.

Thus, once the residue class of $z(\bmod d)$ is fixed, its residue modulo $\operatorname{lcm}(d, 18)$ is determined. Note also that conditions (ii) and (iii) are efficient to test for $p=2$.

However, even with these optimizations there are $\gg B \log B$ pairs $d, z$ satisfying the first line of (5) and conclusions (i) and (iv) of the lemma. To achieve better than $O(B \log B)$ running time therefore requires eliminating some values of $z$ from the start. We accomplish this with a standard time-space tradeoff. To be precise, set $P=3(\log \log B)(\log \log \log B)$, and let $M=\prod_{5 \leq p \leq P} p$ be the product of primes in the interval [5,P]. By the prime number theorem, we have $\log M=(1+o(1)) P$. If $\Delta$ is a square, then for any prime $p \mid M$ we have

$$
\left(\frac{\Delta}{p}\right)=\left(\frac{3 d}{p}\right)\left(\frac{|z|^{3}-c}{p}\right) \in\{0,1\},
$$


where $c \equiv \epsilon\left(\frac{d}{3}\right) k+\frac{d^{3}}{4}(\bmod M)$. When $\operatorname{lcm}(d, 18) \leq \alpha B / M$, we first compute this function for every residue class $|z|(\bmod M)$, and select only those residues for which (7) holds for every $p \mid M$. By Hasse's bound, the number of permissible residues is at most

$$
\frac{M}{2^{\omega(M /(M, d))}} \prod_{p \mid \frac{M}{(M, d)}}\left(1+O\left(\frac{1}{\sqrt{p}}\right)\right)=\frac{M}{2^{\omega(M /(M, d))}} e^{O(\sqrt{P} / \log P)},
$$

and thus the total number of $z$ values to consider is at most

$$
\begin{gathered}
\sum_{\operatorname{lcm}(d, 18) \leq \frac{\alpha B}{M}} r_{d}(k)\left[M+\frac{e^{O(\sqrt{P} / \log P)}}{2^{\omega(M /(M, d))}} \frac{\alpha B}{d}\right]+\sum_{\substack{d \leq \alpha B \\
\operatorname{lcm}(d, 18)>\frac{\alpha B}{M}}} \frac{r_{d}(k) \alpha B}{d} \\
\ll_{k} B \log M+\frac{e^{O(\sqrt{P} / \log P)}}{2^{\omega(M)}} \sum_{g \mid M} \frac{2^{\omega(g)} r_{g}(k)}{g} \sum_{d^{\prime} \leq \frac{\alpha B}{g g M}} \frac{r_{d^{\prime}}(k) \alpha B}{d^{\prime}} \\
\ll_{k} B \log M+B \log B \frac{e^{O(\sqrt{P} / \log P)}}{2^{\omega(M)}} \prod_{p \mid M}\left(1+\frac{2 r_{p}(k)}{p}\right) \\
\ll B P+\frac{B \log B}{2^{(1+o(1)) P / \log P}} \ll B(\log \log B)(\log \log \log B) .
\end{gathered}
$$

For the $z$ that are not eliminated in this way, we follow a similar strategy with a few other auxiliary moduli $M^{\prime}$ composed of larger primes, in order to accelerate the square testing. We precompute tables of cubes modulo $M^{\prime}$ and Legendre symbols modulo $p \mid M^{\prime}$, so that testing (7) is reduced to table lookups. Only when all of these tests pass do we compute $\Delta$ in multi-precision arithmetic [8] and apply a general square test, and this happens for a vanishingly small proportion of candidate values. In fact we expect the number of Legendre tests to be bounded on average, so in total, finding all solutions with $|z| \leq B$ should require no more than $O_{k}(B(\log \log B)(\log \log \log B))$ table lookups and arithmetic operations on integers in $[0, B]$.

Thus, when $B$ fits within the machine word size, we expect the running time to be nearly linear, and this is what we observe in practice for $B<2^{64}$.

\section{Implementation}

We implemented the above algorithm in $\mathrm{C}$, with a few inline assembly routines for Montgomery arithmetic [14] written by Buhrow [4], and Walisch's primesieve library [19] for enumerating prime numbers.

The algorithm is naturally split between values of $d$ with a prime factor exceeding $\sqrt{\alpha B}$ and those that are $\sqrt{\alpha B}$-smooth. The former set of $d$ consumes more than two-thirds of the running time, but is more easily parallelized. We ran this part on the massively parallel cluster Bluecrystal Phase 3 at the Advanced Computing Research Centre, University of Bristol. For the smooth $d$ we used a separate small cluster of 32- and 64-core nodes.

We searched for solutions to (1) for $k$ as in (2) and $\min \{|x|,|y|,|z|\} \leq 10^{16}$, and found the following:

$$
\begin{aligned}
& 33=8866128975287528^{3}+(-8778405442862239)^{3}+(-2736111468807040)^{3}, \\
& 795=(-14219049725358227)^{3}+14197965759741571^{3}+2337348783323923^{3} .
\end{aligned}
$$


We also searched for solutions for $k=3$, addressing a question of Mordell [16, Sect. 6]. In this case, Cassels [5] observed that cubic reciprocity forces the additional constraint $x \equiv y \equiv z(\bmod 9)$, and it follows that part (i) of the lemma can be upgraded to a congruence modulo 162:

$$
z \equiv 4\left(\frac{d}{3}\right) d+3\left(d^{2}-1\right) \quad(\bmod 162)
$$

Despite this added efficiency, we found no solutions, beyond the known single-digit solutions, with $\min \{|x|,|y|,|z|\} \leq 10^{16}$.

The running time was approximately 8 core-years per number tested.

Acknowledgements

I thank Roger Heath-Brown for helpful comments and suggestions.

Received: 15 May 2019 Accepted: 22 June 2019 Published online: 19 July 2019

\section{References}

1. Beck, M., Pine, E., Tarrant, W., Yarbrough Jensen, K.: New integer representations as the sum of three cubes. Math. Comput. 76(259), 1683-1690 (2007)

2. Bosma, W., Cannon, J., Fieker, C., Steel, A.: Handbook of Magma Functions, 2.24th edn. University of Sydney, Sydney (2018)

3. Bremner, A.: On sums of three cubes, Number theory (Halifax, NS, 1994). In: Conf. Proc., vol. 15, Amer. Math. Soc., Providence, pp. 87-91 (1995)

4. Buhrow, B.: YAFU, (2019). https://sourceforge.net/projects/yafu/

5. Cassels, J.W.S.: A note on the Diophantine equation $x^{3}+y^{3}+z^{3}=3$. Math. Comput. 44(169), $265-266$ (1985)

6. Elkies, N.D.: Rational points near curves and small nonzero $\left|x^{3}-y^{2}\right|$ via lattice reduction, Algorithmic number theory (Leiden, 2000), Lecture Notes in Comput. Sci., vol. 1838, Springer, Berlin, , pp. 33-63 2000)

7. Elsenhans, A.S., Jahnel, J.: New sums of three cubes. Math. Comput. 78(266), 1227-1230 (2009)

8. Granlund, T.: The GMP development team, GNU MP: The GNU Multiple Precision Arithmetic Library, 6.1.2 ed., (2016). https://gmplib.org/

9. Heath-Brown, D.R.: The density of zeros of forms for which weak approximation fails. Math. Comput. 59(200), 613-623 (1992)

10. Heath-Brown, D.R., Lioen, W.M., te Riele, H.J.J.: On solving the Diophantine equation $x^{3}+y^{3}+z^{3}=k$ on a vector computer. Math. Comput. 61(203), 235-244 (1993)

11. Huisman, S.G.: Newer sums of three cubes, (2016). arXiv:1604.07746

12. Koyama, K., Tsuruoka, Y., Sekigawa, H.: On searching for solutions of the Diophantine equation $x^{3}+y^{3}+z^{3}=n$. Math. Comput. 66(218), 841-851 (1997)

13. Miller, J.C.P., Woollett, M.F.C.: Solutions of the Diophantine equation $x^{3}+y^{3}+z^{3}=$ k. J. Lond. Math. Soc. 30, 101-110 (1955)

14. Montgomery, P.L.: Modular multiplication without trial division. Math. Comput. 44(170), 519-521 (1985)

15. Montgomery, P.L.: Speeding the Pollard and elliptic curve methods of factorization. Math. Comput. 48(177), 243-264 (1987)

16. Mordell, L.J.: On the integer solutions of the equation $x^{2}+y^{2}+z^{2}+2 x y z=n$. J. Lond. Math. Soc. 28, 500-510 (1953)

17. Niven, I., Zuckerman, H.S., Montgomery, H.L.: An Introduction to the Theory of Numbers, 5th edn. Wiley, New York (1991)

18. The PARI Group, Univ. Bordeaux, PARI/GP version 2 . 11 . 0, (2018). http://pari.math.u-bordeaux.fr/

19. Walisch, K.: primesieve, (2019). https://primesieve.org

\section{Publisher's Note}

Springer Nature remains neutral with regard to jurisdictional claims in published maps and institutional affiliations. 